\title{
SOH Estimation of Lithium-Ion Battery Pack Based on Integrated State Information from Cells
}

\author{
Xiaohong Wang ${ }^{1}$, Wenhui Fan ${ }^{2}$, Shixiang $\mathrm{Li}^{1}$, Xinjun $\mathrm{Li}^{3}$ and Lizhi Wang ${ }^{4, *}$ \\ 1 School of Reliability and Systems Engineering, Beihang University, Beijing 100191, China; \\ wxhong@buaa.edu.cn (X.W.); lishixiang@buaa.edu.cn (S.L.) \\ 2 School of Mechanical Engineering and Automation, Beihang University, Beijing 100191, China; \\ fanwenhui@buaa.edu.cn \\ 3 Asia-Pacific Space Cooperation Organization, Beijing 100070, China; lixinjun@apsco.int \\ 4 Institute of Unmanned System, Beihang University, Beijing 100191, China \\ * Correspondence: wanglizhi@buaa.edu.cn; Tel.: +86-010-8231-7399
}

Received: 26 August 2020; Accepted: 21 September 2020; Published: 23 September 2020

\begin{abstract}
Accompanied by the development of new energy resources, lithium-ion batteries have been used widely in various fields. Due to the significant influence of system performance, much attention has been paid to the accurate estimation and prediction about health status of lithium-ion batteries. In a battery pack, the structure connection causes sophisticated interaction between cells, or between the cells and the pack. Therefore, the degradation of any cell is the result of the deterioration of conjoint cells, and a rapid degradation speed for any individual cell can lead to the accelerated degradation of others beyond expectation, which is one of the primary reasons why the State of Health and life cannot be calculated precisely. To solve this problem, a novel method based on integrated state information from cells has been proposed to estimate status of packs, considering about the degradation effect that cells contribute to the corresponding pack. Using this method, the interactive relationship was described in the form of a neural network in order to mine the effect from the inter-degradation between cells. It was proven that the novel method had better performance than a method based only on the degradation indicators from battery packs.
\end{abstract}

Keywords: lithium-ion battery pack; long-short term memory; degradation interaction; state of health

\section{Introduction}

As a kind of eco-friendly energy, lithium-ion batteries have been widely used in electronic products, electric vehicles, and various types of aircraft, with advantages, such as stability, high energy density, long lifetimes, environmental protection, and so on. Being part of a power supply device, in practical use, the system performance decline of these battery packs causes accidents without being monitored. Therefore, the accurate assessment of battery health has become a pressing problem of great concern in practical use.

The voltage of the lithium-ion battery cell is relatively lower, so batteries must be used in combination. However, most studies about the state of health (SOH) assessment and prediction of lithium-ion batteries are focused on the modeling and methods for objects that we can monitor directly. It means that we can only assess and predict by direct indicators without indirect factors, such as status estimation of cells [1,2], fault diagnosis tools development [3], remaining useful life (RUL) prediction [4]. For example, in recent studies, the analysis on packs only consider about the indicators captured from the packs, including total current, total voltage.

But the problem is that due to the correlation of the electrical connections, the interaction between cells is widespread [5], which means the irregularly degradation process of one cell will cause other 
cells to deteriorate rapidly [6]. Therefore, the overall performance of a battery pack depends on the comprehensive state of all cells in the structure $[7,8]$. Based on this premise, the degradation of a battery pack is essentially the result of the interaction of a large number of cells. Each characteristic of the cells or other items in the pack makes sense for assessment and prediction of the pack, which is always researched as dependency between cells and packs in recent studies.

The typical dependency analysis methods that are widely used at present are divided into two classes: the statistical approach represented by Copula [9] and the probability graph model (PGM) [10] represented by a Bayesian network. However, it has been found that there are evident disadvantages for these two classes:

- The algorithm complexity of a copula is relatively high [11], especially for high-dimension extension problems, such as analysis for battery packs with a great number of cells [12]. Similarly, linear or nonlinear correlation functions that are used to quantize the interaction cannot be extended easily either [13].

- For PGMs, the Bayesian network and the Markov process take advantage of graph structures [14]. Additionally, due to the complication of electrochemical properties, there are many obstacles to establish chemical reaction models for battery packs.

Hence, a method that can effectively integrate state information of cells for pack evaluation is needed. Therefore, we aim at expressing the health sate of the whole pack with dependency information from the cells for more practical application. Compared with traditional dependency analysis methods mentioned above, in recent years, data-driven methods shows excellent merits in engineering applications [15]. Modified algorithms based on Kalman filters [16], particle filters, support vector regression [17], neural networks [18], and fuzzy logic also have been applied to the $\mathrm{SOH}$ analysis of battery cells. Among these methods, neural networks have been developing the most rapidly in recent years, with strong advantages. For example, Mohsen et al. established a novel prediction model of lithium-ion battery cycle life using a feed-forward neural network [19]. Farzaneh et al. used a neural network to achieve the short-term power prediction of a battery [20].

Considering about the most critical step for achieving the expression of the interactive degradation within battery packs is how to make use of the multi-degradation information for the system description, the high-dimension data processing is the most significant problem. In this area, deep learning shows strong advantages [21]. Time series analysis models, such as recurrent neural network (RNN), do well in time-dependent relations model realization [22]. The historical data memory and relationship analysis ability of an RNN can be utilized to screen and retain informative the degradation process from the long-term data. With the help of deep learning, this type of network is expected to achieve a model description of the multi-degradation process and an accurate $\mathrm{SOH}$ prediction [23]. At present, neural networks have been increasingly used in $\mathrm{SOH}$ prediction and estimation of batteries.

Take advantage of deep learning, in this research, we adopted a data-driven theory to propose a multi-cell degradation information fusion method for battery pack $\mathrm{SOH}$ prediction based on deep learning, making full use of the inter-degradation information of the cells. In the proposed method, there are three critical improvements to achieve our goal:

1. Different with the existing studies, the status of battery pack is the object focused on to realize global assessment result of the power system instead of $\mathrm{SOH}$ of cells.

2. Multi-dimension SOH data of cells from different levels of structure are utilized as input to assess battery packs, instead of only pack indicators, which provide dependency information in interior.

3. To improve prediction performance, not only $\mathrm{SOH}$ information, but also exhaustive temperature data of battery cells is captured to offer status information of battery cells.

To verify the performance and feasibility of the novel method, the experiments were carried out for battery packs. In the experiment, the electrical indicators of cells and packs were acquired as input for the model established based on long-short term memory (LSTM) basic structure and they were used to determine the interaction between the cells for $\mathrm{SOH}$ prediction. 
The main content of this paper is arranged as follows: Section 2 briefly introduces the basic method theories involved in this paper. Based on the basic theories, the main ideas of the technical method proposed in this paper is explained in Section 2. As described in Section 3, for the demands of $\mathrm{SOH}$ data collection, the cycle experiments of battery packs were designed for data acquisition. The data for typical battery packs were preliminary analyzed for subsequent research and the validation of the method. In Section 4, the data set collected in the experiments mentioned in Section 4 is used to verify the feasibility and advantages of the novel method. Section 5 summarizes the entire paper.

\section{Methodology}

\subsection{Indicators of Battery}

To describe the deterioration process, the electrical parameters during charge and discharge were mostly used generally. For cells, there were numerous indicators used to monitor the status of the batteries, such as the discharge current, cut-off voltage, and open-circuit voltage. The most common assessment indicator of batteries is capacity, which is measured by calculating the electric quantity. The electric quantity of battery is always estimated by ampere-hour method. According to ampere-hour method, the capacity is calculated as:

$$
Q=\int_{0}^{t} \eta I d t
$$

where $Q$ represents the current capacity, $I$ is the discharge current, and $\eta$ is the discharge coefficient. Here are three commonly used: $\mathrm{SOH}$, state of charge (SOC) and depth of discharge (DOD). SOC and DOD are used to assessment the ability at time $t$ under one discharge cycle. DOD is the released electric quantity on a given cycle, expressed as a percentage of the total capacity of the battery:

$$
\mathrm{DOD}=\frac{Q_{\text {released }}}{Q_{\text {rated }}} \times 100 \%
$$

SOC is the verse of DOD, which is defined as [24]:

$$
\operatorname{SOC}(t)=100 \%-\operatorname{DOD}(t)=\frac{Q_{\text {releasable }}}{Q_{\text {rated }}} \times 100 \%,
$$

When the deterioration of battery is not considered, $Q_{\text {rated }}$ is constant. However, in most cases, the rated capacity of battery decreased under charge and discharge cycles. $\mathrm{SOH}$ is used to assess the extent of the reduction of rated capacity. The unit of $\mathrm{SOH}$ is percent, and $100 \%$ means it is a fresh battery [25]. Here we define the failure threshold of battery is $80 \%$ initial capacity. If $Q_{0}$ is assumed to be the initial capacity, then the $\mathrm{SOH}$ of a battery is defined by:

$$
\mathrm{SOH}=\frac{Q}{Q_{0}} \times 100 \%
$$

In our research, $\mathrm{SOH}$ is utilized to present the evolution of different cycles in order to assess health status of cells and battery packs.

\subsection{Neural Network Structure}

Deep learning is an important branch of machine learning technology that is established based on a neural network model. As discussed in the previous part of this paper, compared with statistical theory, probability graph models, and other means, the advantage of machine learning is the ability to analyze large amounts of original data and to extract characteristics [26]. The implementation process of deep learning is illustrated in Figure 1. 


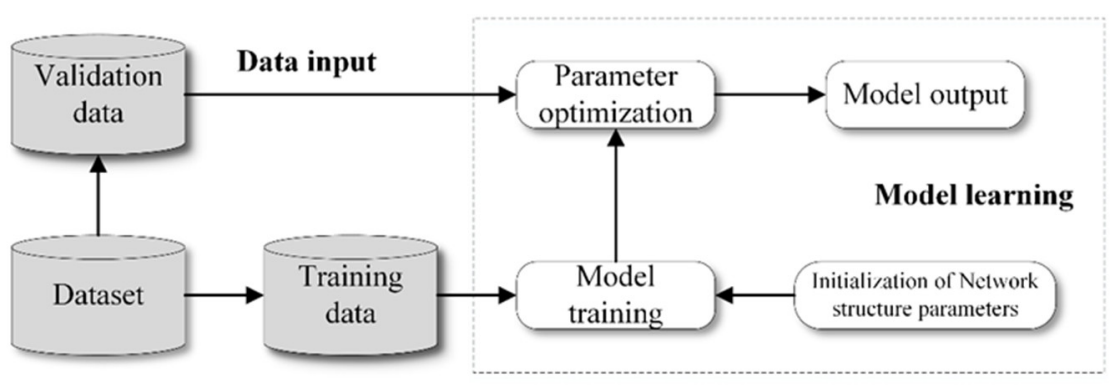

Figure 1. The flowchart of deep learning.

For applications of deep learning, a task involving time series analysis is a typical problem. Currently, many researchers are studying algorithms to analyze the characteristics of a time series. An LSTM is a kind of model structure that has many applications. LSTM was first proposed by Sepp Hochreiter and Jürgen Schmidnuber, and its basic framework was developed from the RNN model for time series processing. As is well known, there is always some long-term memory information in a time series. However, for practical applications of the RNN model, it is difficult to deal with long-term dependence due to the gradient disappearance and gradient explosion in the algorithm. Due to the loss of long-term information, the analysis performance of a time series is very limited. LSTM is an extended RNN model proposed in this context to solve problems involving long calculation times and the forgetfulness of long-term information [27]. LSTM belongs to a gated RNN sequence model, and its principle is shown in Figure 2. Through this type of structure setting, a neural network can remove or increase information memory with the help of a gate structure in order to achieve better dependent information learning. In the following proposed method, LSTM is utilized to be the main structure of integrate model.

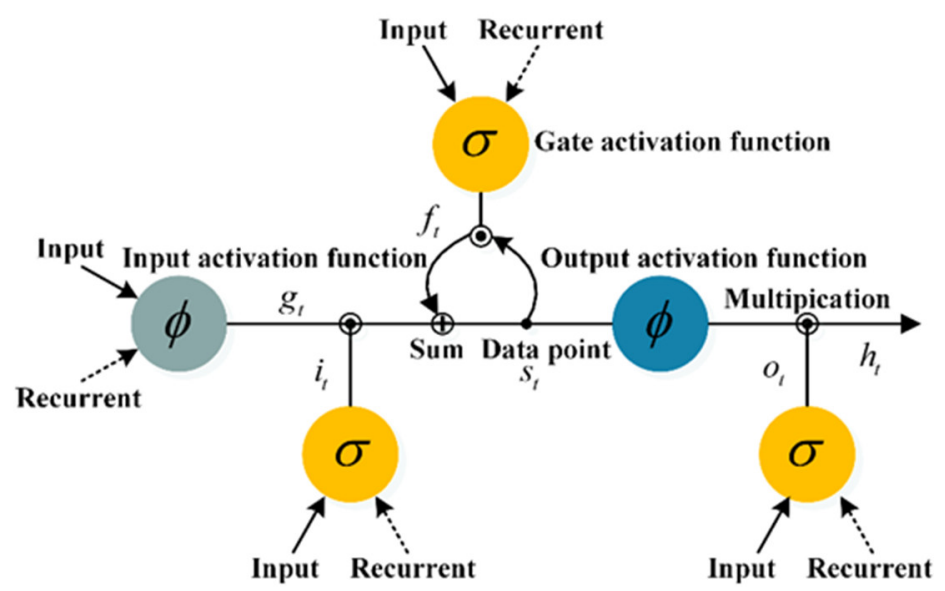

Figure 2. Long-short term memory (LSTM) model.

In the training of neural networks, insufficient data leads to overfitting problems, which refer to the phenomenon for which a model has a fixed memory of the training data. This makes the output performance of training data set excellent, while the performance of validation dataset is extremely poor. To solve overfitting problems, the dropout method was proposed [28]. The core idea of dropout is optimizing the network to be thinner by integrating all of the subnetworks via the removal of non-output units from the primary network (see Figure 3) and reducing the computation burden with the same training parameters. In this research, dropout was adopted to avoid rote memorization of the training dataset. 


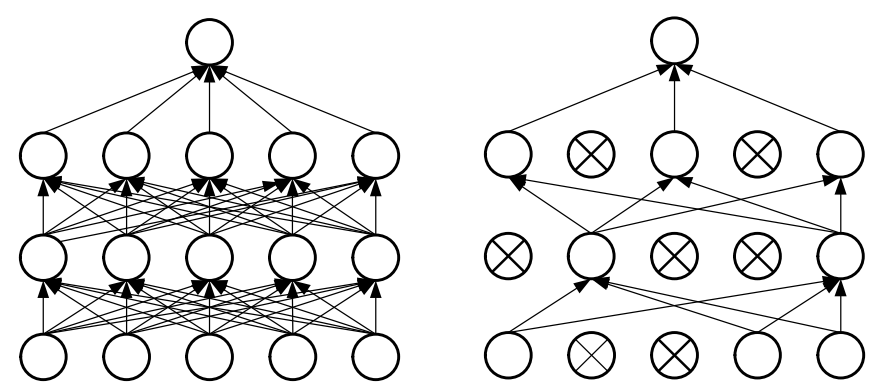

Figure 3. The principle of dropout.

\subsection{Deterioration Information Integrate for $\mathrm{SOH}$ Prediction}

For deterioration information integrate, data collection, processing, prediction and validation are all significant steps. Therefore, before the specific prediction method is given, the whole technological process of deterioration information integrate for $\mathrm{SOH}$ prediction is introduced, as seen in Figure 4 . Firstly, reasonable acquisition method is designed to collect running data. The monitored objects in collection depend on the state data used for prediction and assessment, including cells and packs. In general, voltage, current, running time, sampling frequency and temperature are all necessary parameters for $\mathrm{SOH}$ analysis. After data collection, the original data need to be processed for better performance in following analysis. Secondly, the proposed method is used to predict $\mathrm{SOH}$ of packs based on integrated states and ambient indicators of cells, which is realized by deep learning model (LSTM) and corresponding algorithm, such as regularization and optimization algorithms. At the same time, the deep learning model is also used to predict only based on states and ambient indicators of the packs, which is used to compare. At last, there is a comparison between predictions based on state indicators from cells and packs to validate the effectiveness of information integrate.

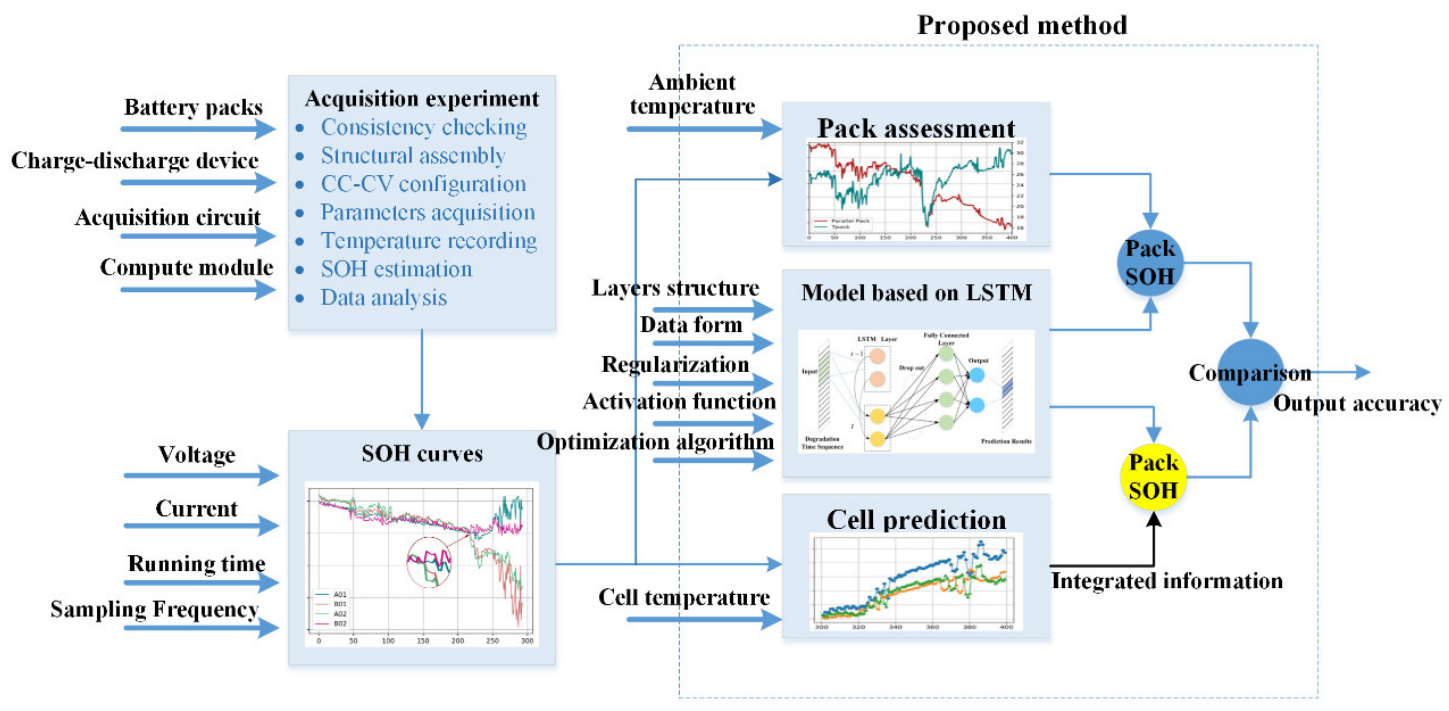

Figure 4. Schematic overview of the whole paper.

Here we will discuss the theoretical method in order to demonstrate the core ideas firstly. In later sections, the experiment and validation will be set forth follow this process.

In this research, the basic framework of LSTM was adopted to build the $\mathrm{SOH}$ prediction model in order to utilize the multi-cell degradation information for interaction to predict the overall $\mathrm{SOH}$ of the battery packs. The flow chart of the proposed method according to the basic structure of the deep learning method is shown in Figure 5. Referring to the framework in Figure 5, the specific process can be explained as follows: 


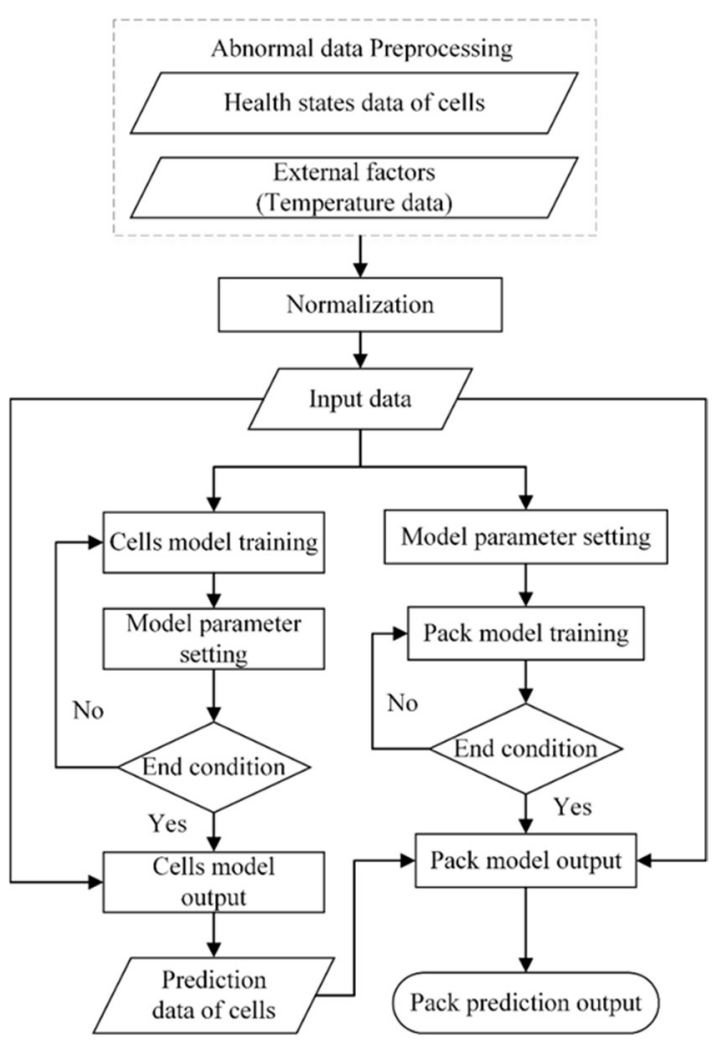

Figure 5. Flow chart of the proposed method.

\subsubsection{Input Data Preparation}

The critical point of overall $\mathrm{SOH}$ prediction is to obtain the degradation trend of cells under interaction. This input information can be learned from the earlier degradation process of the battery cells. If $S_{\text {ori }}$ represents the training dataset collected in the experiment, the normalized dataset can be expressed as $S$. In order to provide a clear description, this paper describes the mathematical expressions under the condition that the health status indicators only include the $\mathrm{SOH}$. The health status data of the cells is expressed as $S_{c}=\left(X_{1}, X_{2}, \cdots, X_{n}, \cdots, X_{N}\right)^{T}, n \in N$, where $N$ is the number of cells located in the battery pack. The health status data of the battery pack is expressed as $S_{P}=\left(Y_{1}, Y_{2}, \cdots, Y_{m}, \cdots, Y_{M}\right)^{T}, m \in M$, where $M$ represents the indicator types of the battery pack. The simplified indicator expression can be written as $S_{P}=[Y]$. The health status data $X_{n}$ of each battery cell can be expressed as a high-dimensional time series $X_{n}=\left(\begin{array}{c}X_{1, n} \\ X_{2, n} \\ \vdots \\ X_{p, n}\end{array}\right), p \in P$ represents the types (dimensions) of the health status indicators. $X_{p, n}=\left(x_{p, 1}^{n}, x_{p, 2}^{n}, \ldots, x_{p, t}^{n}, \ldots, x_{p, T}^{n}\right)$ is the captured data for the $p$ th indicator. $t \in\{1,2, \ldots, T\}$ is the time moments and $T$ is the collection time. Thus, the input data and the output data structure in this model were chaired. However, considering the generally poor prediction performance of a time series, a large error may have been introduced in the input link. The time label and the ambient temperature were used as auxiliary input data to help estimate the $\mathrm{SOH}$.

- Time label: For the circumstance of no external impact, the degradation process of battery cells is consistent. The main deterioration of battery cells can be determined by taking the number of cycles as an estimation benchmark. Here, the time label input is expressed as $L=\left(l_{1}, l_{2}, \ldots, l_{T}\right)$. Similarly, $T$ is the collection time of the time series. 
- Ambient temperature: A slight fluctuation of the ambient temperature has an influence on the charge and discharge performance of battery cells. Consideration of the ambient temperature improves the prediction accuracy to a certain extent. Here, the environmental temperature input is expressed as $D_{\text {temp }}=\left(d_{1}, d_{2}, \ldots, d_{t}, \ldots, d_{T}\right)$.

Based on the above description, it can be concluded that the input of the cells prediction model was $X_{\text {input }}^{c}=\left(\begin{array}{c}S_{c} \\ L \\ D_{\text {temp }}\end{array}\right)$, and the input of the pack prediction model was $X_{\text {input }}^{p}=\left(\begin{array}{c}S_{p} \\ L \\ D_{\text {temp }}\end{array}\right)$. The input mode of the training data for the pack model is shown in Figure 6.

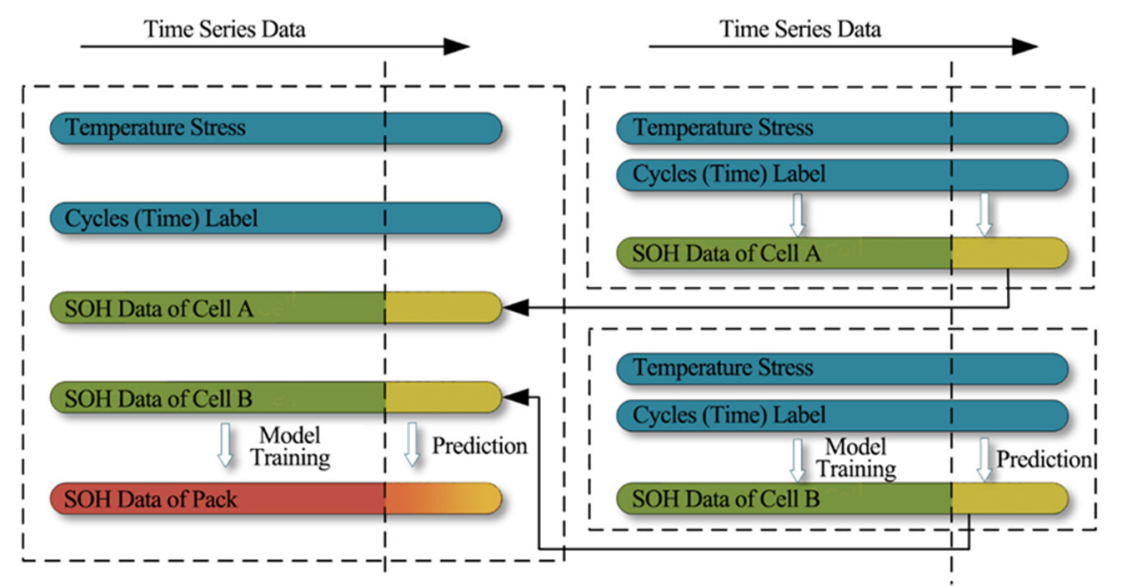

Figure 6. Structure and allocation of input data.

\subsubsection{Network Model Building}

The determination of a network structure depends on dataset form, scale, and optimization result. The network structure proposed in this paper was based on LSTM and the dropout method. This structure is shown in Figure 7.

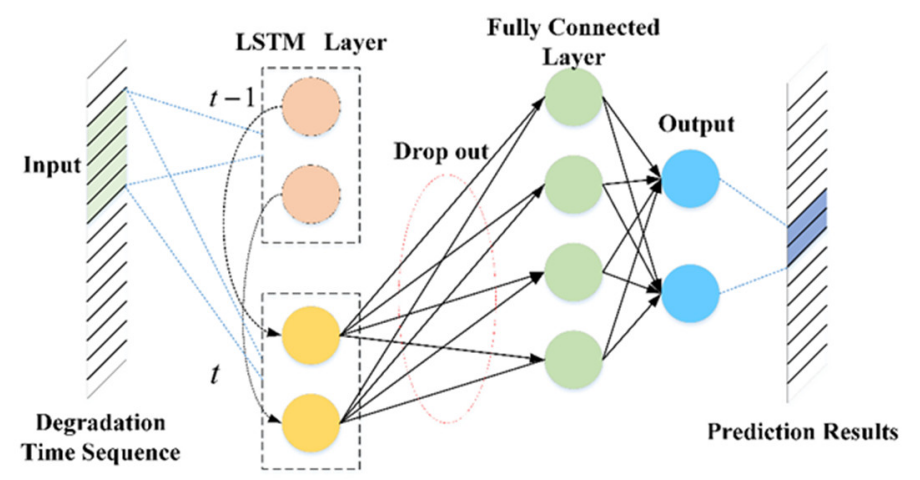

Figure 7. Network model for the pack state of health $(\mathrm{SOH})$ prediction.

- Input layer: The degradation time series of battery cells is taken as the model input, whose dimension depends on the number of cells located in the battery pack and on the auxiliary indicator parameters (time label, ambient temperature, etc.). In this research, there is only one health status type, and $p=1$. Hence, subject to the battery pack structure in the experiment, $N=2$ in series and parallel packs, and the input data were set as a matrix including four vectors: 


$$
X_{\text {input }}^{c}=\left(\begin{array}{c}
S_{c} \\
L \\
D_{\text {temp }}
\end{array}\right)=\left(\begin{array}{cccccc}
x_{1,1}^{1} & x_{1,2}^{1} & \ldots & x_{1, t}^{1} & \ldots & x_{1, T}^{1} \\
x_{1,1}^{2} & x_{1,2}^{2} & \ldots & x_{1, t}^{2} & \ldots & x_{1, T}^{2} \\
l_{1} & l_{2} & \ldots & l_{t} & \ldots & l_{T} \\
d_{1} & d_{2} & \ldots & d_{t} & \ldots & d_{T}
\end{array}\right)
$$

Further, for the series-parallel pack, it is a matrix including six vectors with $N=4$ :

$$
X_{\text {input }}^{c}=\left(\begin{array}{c}
S_{c} \\
L \\
D_{\text {temp }}
\end{array}\right)=\left(\begin{array}{cccccc}
x_{1,1}^{1} & x_{1,2}^{1} & \ldots & x_{1, t}^{1} & \ldots & x_{1, T}^{1} \\
x_{1,1}^{2} & x_{1,2}^{2} & \ldots & x_{1, t}^{2} & \ldots & x_{1, T}^{2} \\
x_{1,1}^{3} & x_{1,2}^{3} & \ldots & x_{1, t}^{3} & \ldots & x_{1, T}^{3} \\
x_{1,1}^{4} & x_{1,2}^{4} & \ldots & x_{1, t}^{4} & \ldots & x_{1, T}^{4} \\
l_{1} & l_{2} & \ldots & l_{t} & \ldots & l_{T} \\
d_{1} & d_{2} & \ldots & d_{t} & \ldots & d_{T}
\end{array}\right)
$$

Considering the difficulty and accuracy of the auxiliary indicators, the time and ambient temperature, which were easy to monitor, were selected to reduce the accumulated errors caused by degradation fluctuation.

- Hidden layer: The internal structure of this model was composed of several LSTM network structures, and the number of layers and neurons depended on the data dimension and scale. With increase of quantity and scale, a neural network composed of only LSTM is prone to experiencing overfitting, which makes the generalization ability of the model extremely poor. In order to reduce the occurrence of overfitting, dropout was set between every two LSTM layers, and the neurons were randomly discarded and reconstructed.

- Output layer: The output model was the degradation indicator time series of the battery pack, and its dimension was consistent with the input sequence. The output was the $\mathrm{SOH}$ prediction values of the battery pack.

Accordingly, the model for deterioration information integrate, which is used to predict status of packs, has been built. The model extracts numerical characteristics of cells related to pack degradation, and expresses the function relations between them to integrate all deteriorate information in cells.

\subsubsection{Hyper-Parameter Solution}

In the process of model training, the setting of the Loss function was a significant link for the model solution. In this research, the minimum MSE (mean-square error) was adopted as the optimization objective. The specific calculation formula of the MSE is:

$$
\gamma=\frac{1}{n} \sum_{i=1}^{n}\left(X_{i}-\hat{X}_{i}\right)^{2}
$$

where $X_{i}$ is the observed real sample sequence and $\hat{X}_{i}$ is the prediction sample sequence of $X_{i}$. The MSE between them can be obtained as $\gamma$ according to the above question. The determination of the model focused on accurately finding the optimal value of the hyper-parameter, which had a direct effect on whether the appropriate model can perform well enough for the dataset. When model training is carried out for the case of a non-convex function, the learning trajectory needs to experience different types of structures and to finally find a region whose local part is a convex bowl. In the past, for such problems, an adaptive gradient algorithm (AdaGrad) algorithm was used to contract the learning rate according to the whole history of the square gradient, but it could make the learning rate reach too small before the convex structure was found. Therefore, the root mean square prop optimizer (RMSprop) algorithm was utilized in this research to optimize the parameters. 
The RMSprop algorithm adopts an exponential decay average to forget the previous learning rate history, enabling the algorithm to rapidly converge after finding the target convex structure. The latest gradient value is used to normalize the current gradient value, and it divides the current gradient by the moving average of the RMS gradient [29]. At first, the learning rate at current time is obtained by Equation (8) as follows:

$$
f^{\prime}\left(\theta_{t}\right)=\frac{\partial L\left(\theta_{t}\right)}{\partial \theta_{t}}
$$

where $L(\theta)$ is the loss function of the optimization problem, which quantitative the consistency of model output and correct results. $\theta$ is parameters of the loss function, influencing the variation characteristics of $L(\theta) . f^{\prime}\left(\theta_{t}\right)$ is the derivative of the loss function $L(\theta)$ with respect to the parameter $\theta$ at moment $t$.

Secondly, the variation $r_{t}$ at moment $t$ is calculated by:

$$
r_{t}=(1-\beta) f^{\prime}\left(\theta_{t}\right)^{2}+\beta r_{t-1}
$$

where $\beta$ is the forgetting factor, typically 0.9 [30]. $\beta$ is a decay term which controls the influence from history information. By Equation (9), dynamic variable is introduced to learning rate of RMSprop.

At last, the derivative can be updated by the following formulas:

$$
\begin{gathered}
v_{t+1}=\frac{\alpha}{\sqrt{r_{t}}} f^{\prime}\left(\theta_{t}\right) \\
\theta_{t+1}=\theta_{t}-v_{t+1}
\end{gathered}
$$

where $v_{t+1}$ is moving step of updating parameter $\theta$, which is decided by step size $\alpha$ and decay term $\beta$. Step size $\alpha$ decides the searching speed of parameter updating. By Equation (11), the learning process of RMSprop is changed.

\subsubsection{Model Training and Output}

Model training is the most critical link for determining the validity and accuracy of a neural network model. In order to obtain satisfying training results in the application scenario described in this paper, the following content needed to be well implemented:

- Normalization: Good normalization was necessary for model training. In the application scenario of this paper, in order to efficiently produce the solution of the parameters, the time label and the ambient temperature are transformed into the same numerical magnitude and uniformly normalized.

- Dataset division: According to the scale of the data obtained from the experiment, the training dataset, validation dataset, and test dataset were divided with the ratios of 8:1:1 or 7:2:1. These division ratios were considered to provide sufficient training data for the optimization of the model parameters, and a validation dataset no smaller than the test set was used to ensure the training performance. It followed the general rules of dataset division of deep learning.

- Data ordering: according to the consistency of characteristics before and after, reordering the data was helpful for including various characteristics in the training data.

After the input data preprocessing, the model was trained. During training, the specific model parameters were determined according to the performance of the training data. The output of model was set as the $\mathrm{SOH}$ of the battery pack.

\section{Experiment and Primary Analysis}

\subsection{Experiment Design}

Due to the complexity and difficulty of monitoring on industrial battery packs, an experiment for battery $\mathrm{SOH}$ prediction was developed and conducted in our study in order to validate the method 
proposed in Section 2. In the experiment, typical structures of battery packs were set up for diverse data, and well-matched equipment were designed to collect running data. In the experiments, 18,650 lithium-ion batteries (Type: ICR18650-22PM) were utilized to conduct the experiment. These batteries were produced by Samsung (Seoul, South Korea). The 18,650 lithium-ion battery is one of the most popular battery types, widely used in various rechargeable electrical products, such as vehicles. The indicators of an ICD18650-22PM lithium-ion battery are listed in Table 1. The consistency of all samples used in the experiment need to be ensured. The samples used in our experiment are referred to as S01-S08 in this paper.

Table 1. Indicators of an ICR18650-22PM lithium-ion battery.

\begin{tabular}{cc}
\hline Item & Specification \\
\hline Cathode Material & $\mathrm{LiCoO}_{2}$ \\
Anode Material & Graphite \\
Rated Capacity & $2.15 \mathrm{Ah}$ \\
Nominal Voltage & $3.62 \mathrm{~V}$ \\
Standard Discharge Current & $1 \mathrm{C}$ \\
Max. Charge Current & $1 \mathrm{C}$ \\
Charging Cut-off Voltage & $4.20 \mathrm{~V} \pm 0.05 \mathrm{~V}$ \\
Max. Discharge Current & $3 \mathrm{C}$ \\
Discharge Cut-off Voltage & $2.75 \mathrm{~V}$ \\
\hline
\end{tabular}

In this experiment, measurement equipment was used to capture and record the charge and discharge states of battery packs. Due to the limited capacity of the measurement equipment, the current and voltage of the cells located in the packs could not be measured directly. In order to test the cells at the same time, we designed a peripheral circuit to monitor the current and voltage of the cells. The schematic diagram of the test platform is shown in Figure 8. As demonstrated in Figure 8, the test platform is divided into two parts. Computer A was responsible for data collection and control of cell screening and pack testing. This part was carried out by battery testing system from Neware Company (Shenzhen, China). Computer B was used to conduct data acquisition of cells testing. Cells testing consists of four similar acquisition models, which have the ability to measure and record current and voltage data. The specific pictures about the platform is introduced by Zhuo Wang [9], and here we will not go into details to avoid redundancy.

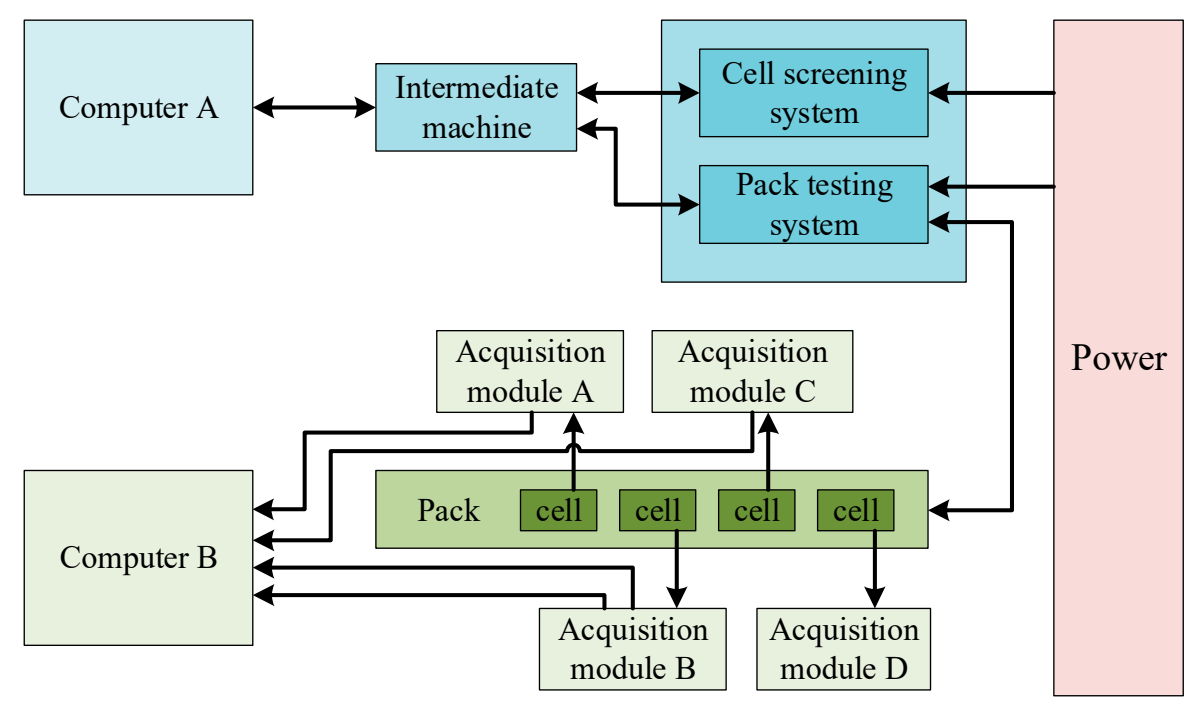

Figure 8. Schematic diagram of the battery pack experiment platform. 


\subsection{Data Acquisition and Analysis}

\subsubsection{Data Acquisition}

The eight cell samples picked from screening were divided into four groups, for which different battery packs were built. The details of the four structures of tested battery packs are listed in Table 2 .

Table 2. Battery pack and cell numbers.

\begin{tabular}{cccc}
\hline Pack Structure & Series & Parallel & Series-Parallel \\
\hline Cells & S01, S02 & S03, S04 & S05, S06, S07, S08 \\
\hline
\end{tabular}

To maintain consistency with a real working environment, the experiments were carried out at room temperature, which means the environment was not controlled during experiments. However, the room temperature was monitored continuously to protect the batteries from thermal shock that might cause inner damage to the cells. Simultaneously, the real-time surface temperatures of the cells were supervised by temperature sensors to provide precise health statuses. The constant current-constant voltage (CC-CV) method was employed to test all of the battery packs.

\subsubsection{Primary Analysis}

Taking a series-parallel pack as an example, the capacity time series data of the pack and cells (S05 S08) can be seen in Figure 9. On the one hand, just as seen in Figure 9, the deterioration of the batteries is related to the charge and discharge cycles. On the other hand, compared with Figure 10, preliminary conclusions can be drawn that the slight fluctuations of degradation are closely related to the environment temperature in a qualitative fashion.

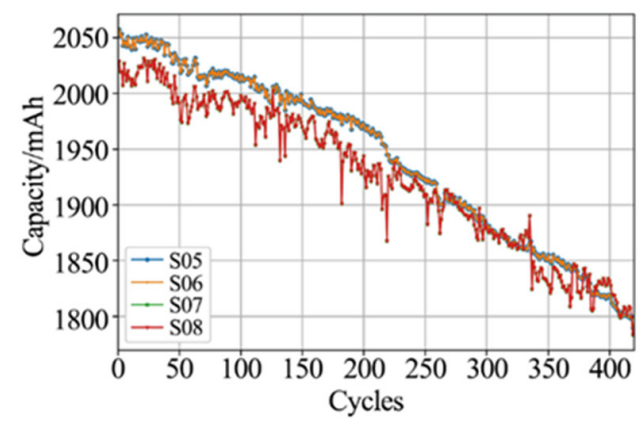

(a)

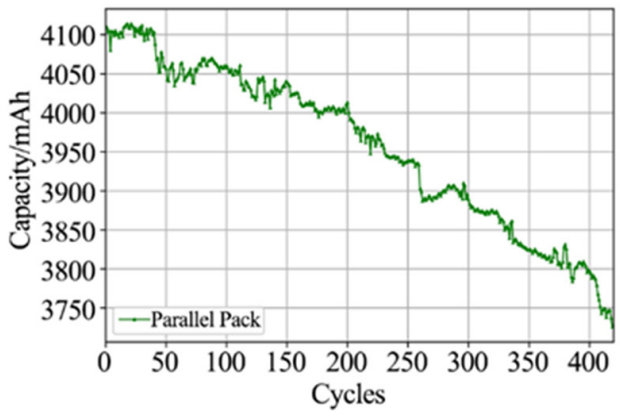

(b)

Figure 9. Capacity degradation curves of a series-parallel pack: (a) cells; (b) pack.

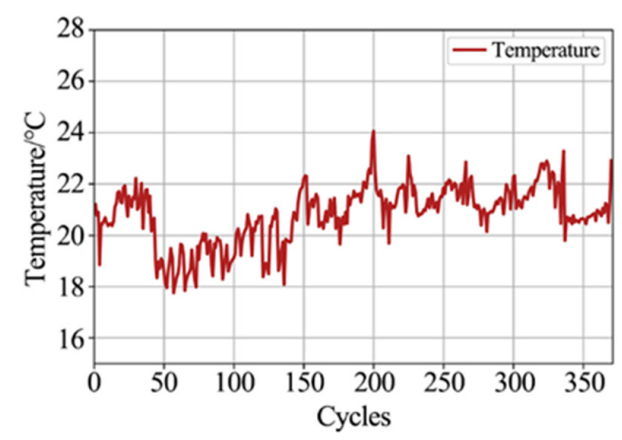

(a)

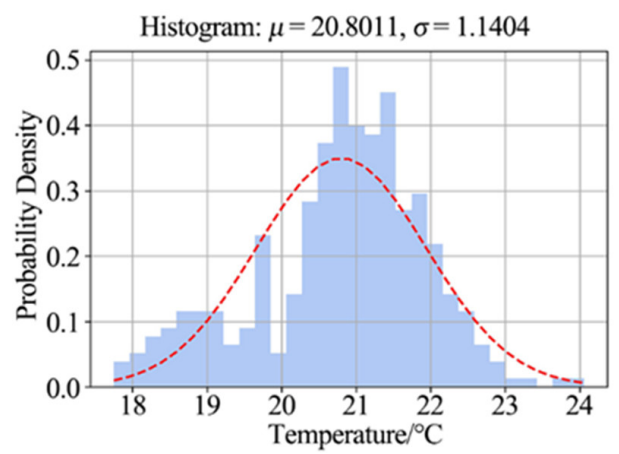

(b)

Figure 10. Environment temperature during experiment: (a) temperature time series; (b) temperature distribution. 
We further ensured the relevance of the degradation rate and temperature using a linear correlation analysis. In the same way, a series-parallel pack was chosen as an example. Figure 11 presents the correlation between the deterioration rates and temperature fluctuation at different times. Taking the performance and the access to input data into account, the time label (cycles) and the temperature were both selected to be input indexes for prediction.

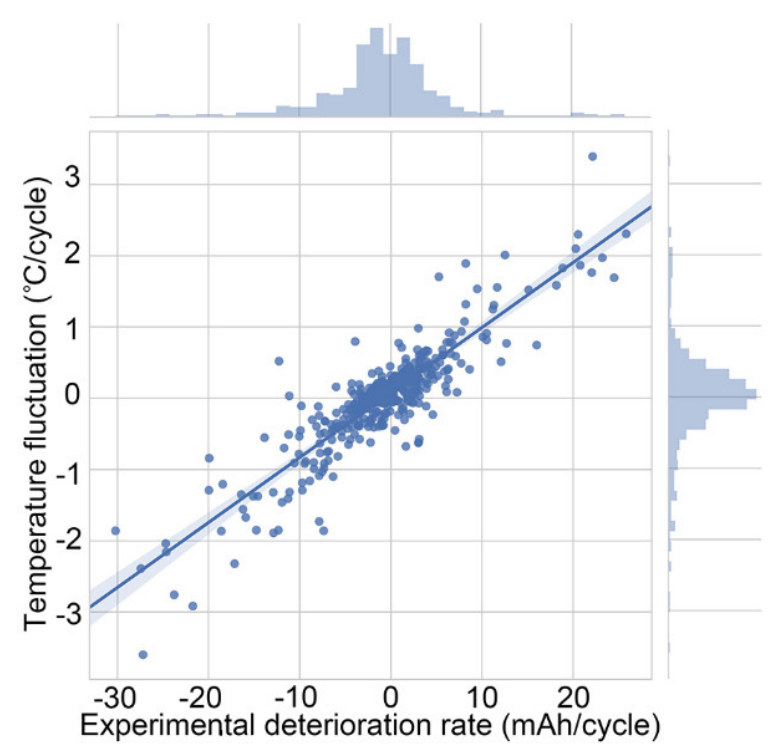

Figure 11. Correlation analysis between the deterioration rate and the temperature fluctuation.

\section{Prediction Result Analysis}

\subsection{Two-Cell Pack Prediction}

For a better analysis of the performance of the proposed method, the validation started from the most basic battery structure, a series and parallel pack. Figure 12 displays the samples and measurement circuit for a two-cell pack. To state the advantage of proposed method, we used a prediction result with only pack indicators for comparison. The abbreviations of two methods used for comparison are listed in Table 3.

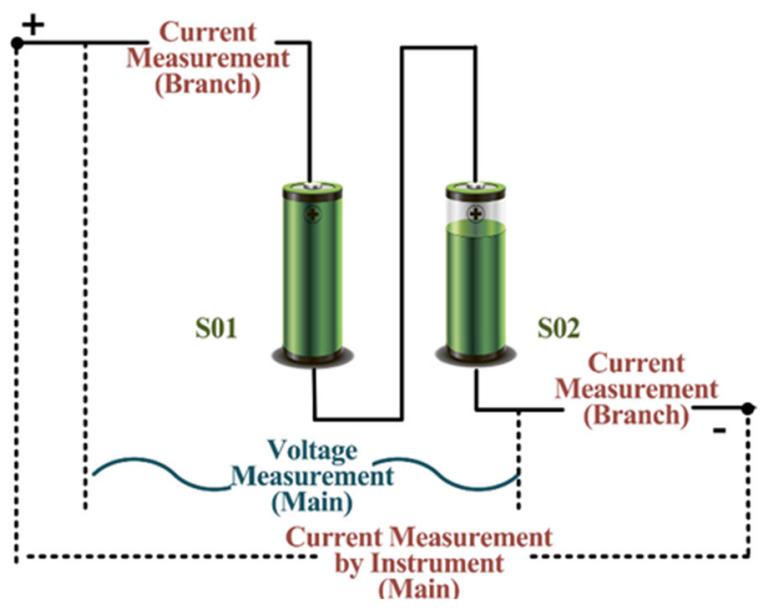

(a)

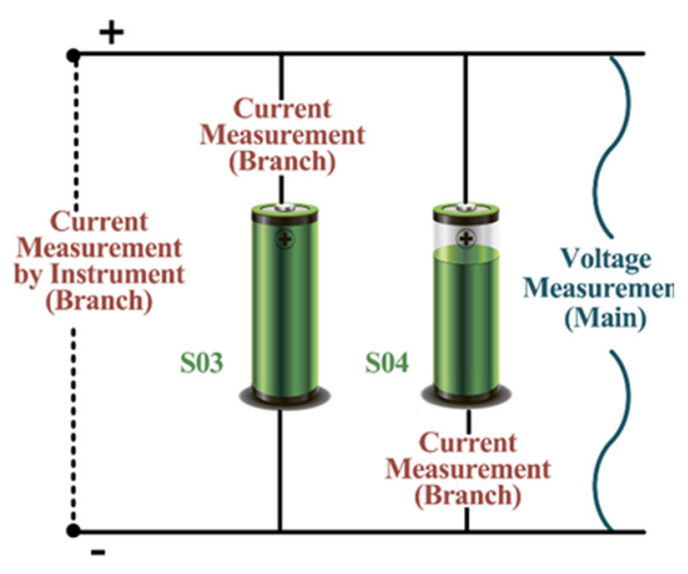

(b)

Figure 12. Two-cell pack measurement schematic diagram: (a) series; (b) parallel. 
Table 3. Abbreviations of the $\mathrm{SOH}$ prediction methods.

\begin{tabular}{ccc}
\hline Abbreviations & Input Data & Output Data \\
\hline Pack-Prediction & $\begin{array}{c}\text { Environment temperature } \\
\text { Cycles (time) label }\end{array}$ & SOH of pack \\
\hline Cells-Prediction & $\begin{array}{c}\text { Environment temperature } \\
\text { Cycles (time) label } \\
\text { SOH prediction data of cells }\end{array}$ & SOH of pack \\
\hline
\end{tabular}

For the simpler structure of series and parallel battery packs, the data fluctuations and rules are relatively stable. Therefore, the prediction performance is excellent in the entire life. The comparison results of the series and parallel pack are demonstrated in Figure 13. For accurate presentation, it only shows the $\mathrm{SOH}$ prediction results of different methods at 341-371 cycles except for the training and test data, and emphasizes the error in prediction phases. In addition, because of the disparity between the experiment times, the cycles of the series and parallel pack tests were slightly different.

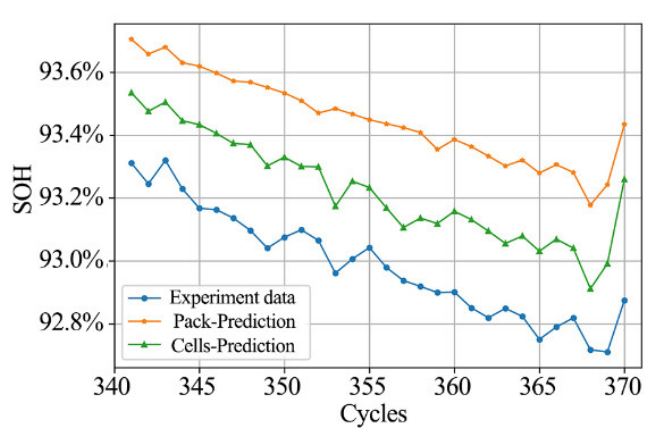

(a)

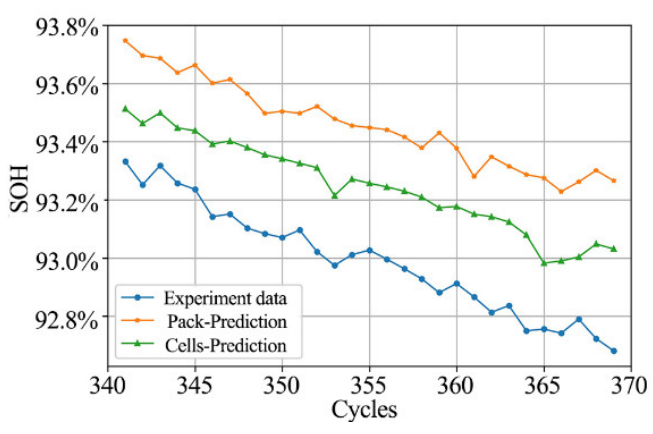

(b)

Figure 13. Comparison of the $\mathrm{SOH}$ prediction results: (a) series; (b) parallel.

Figure 13a,b compare the $\mathrm{SOH}$ of series and parallel packs based on different methods, and same color lines symbolize the same method. As seen in Figure 13, in general, the SOH curves based on pack-prediction and cells-prediction methods are both closed to the real data. It means the both prediction methods have good performance. Furthermore, the cells-prediction always performs better than pack-prediction method. At the same time, the both curves are higher than real experiment data, which means prediction methods always give optimistic life expectation. It is caused by persistent negative impact between cells, which is getting worse during working. For the series pack in Figure 13a, the real data fluctuates apparently, which is captured by cells-prediction method better than the pack-prediction method. The pack-prediction curve is too flat to describe the dynamic degradation process. On the contrary, in the parallel pack, there are too many inaccurate fluctuations in pack-prediction at 345,351 , 359,361 cycles, and so on. It makes the result of pack-prediction unreliable.

For better comparison of the $\mathrm{SOH}$ prediction result, the relative error was adopted to be the indicator showing performances. The relative error was calculated as follows:

$$
\mathrm{RE}=\frac{\hat{y}-y}{y} \times 100 \%
$$

where RE represents the relative error, $\hat{y}$ is the prediction value of the $\mathrm{SOH}$, and $y$ is the real value. The comparison result of the relative errors is shown in Figure 14. 


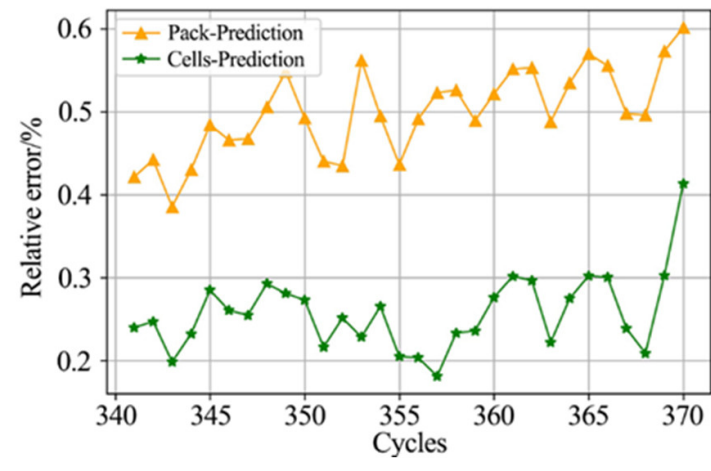

(a)

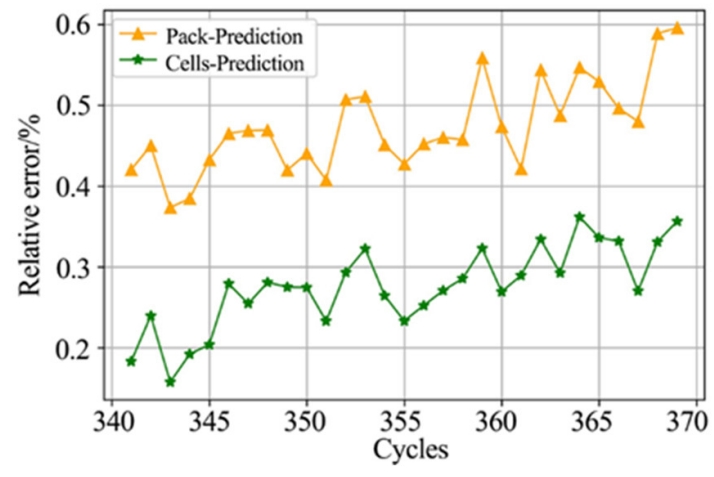

(b)

Figure 14. Relative errors of the prediction results: (a) series; (b) parallel.

The comparison results in Figures 13 and 14 illustrate the fact that the two prediction methods could both achieve high accuracy in the case of a series and parallel structure. It can be seen visually that the cells-prediction method had a more precise and stable performance. However, because of the plain structure, the interaction between cells was limited, which led to the inconspicuous preponderance of the cells-prediction method.

\subsection{Series-Parallel Pack Analysis}

According to the above elaboration, the cells-prediction method could provide better $\mathrm{SOH}$ prediction results under the premise of sufficient information from cells. In order to verify the generalization ability, we provide an application example of a four-cell pack in this section. The structure of a tested pack is shown in Figure 15. Figure 16 shows the comparison of the prediction result.

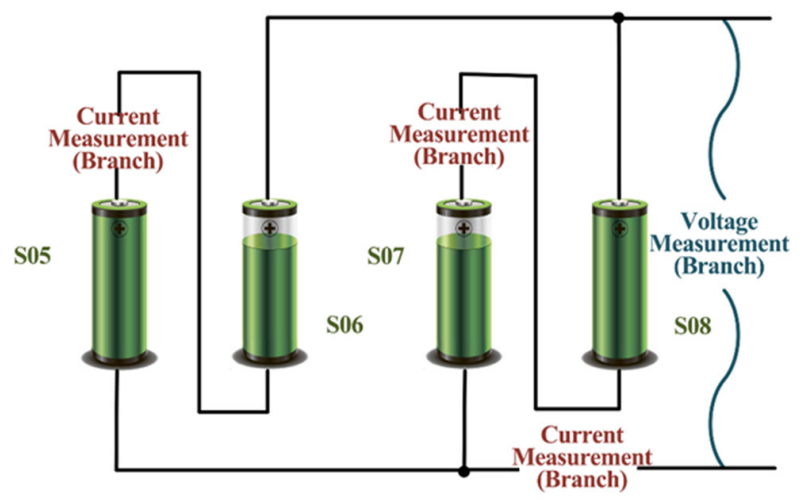

Figure 15. Series-parallel pack measurement schematic diagram.

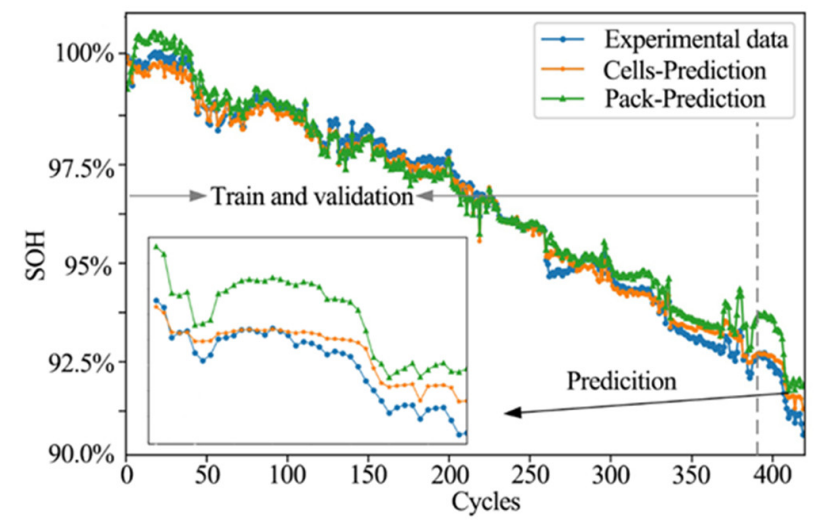

Figure 16. Comparison of the $\mathrm{SOH}$ prediction result for a series-parallel pack. 
Among the 420 cycles data acquired in the experiment, the first 379 cycles ( $90 \%$ of the data) were used to train and construct the model, and the final 42 cycles ( $10 \%$ of data) were used to compare the prediction performance. As shown in Figure 16, in the prediction data, the cells-prediction gave the closest result to the experimental data, for which there were obvious fluctuations in the pack-prediction. Through data analysis, it was realized that the reason for this was a strong dependence on the input data caused by input information being too limited. In other words, when the temperature data appeared to fluctuate, the prediction result of the pack-prediction waved in an exaggerated fashion. To confirm this reason, the correlation analysis between the degradation rate and temperature fluctuation is shown in Figure 17.

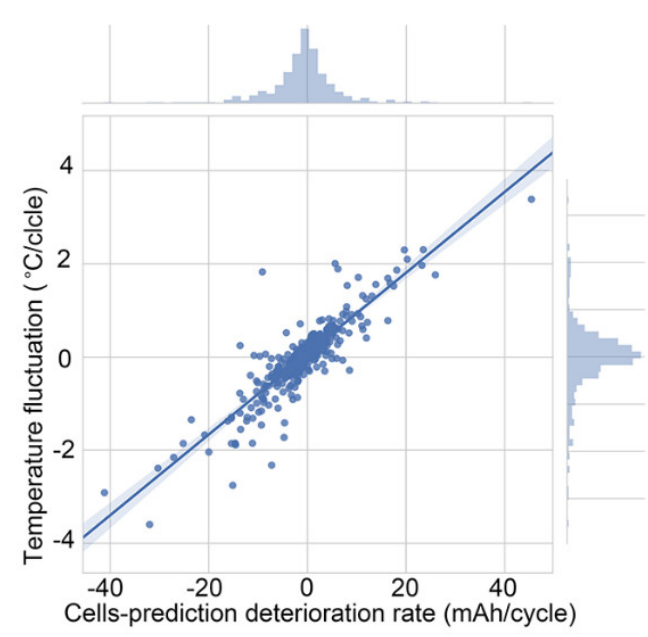

(a)

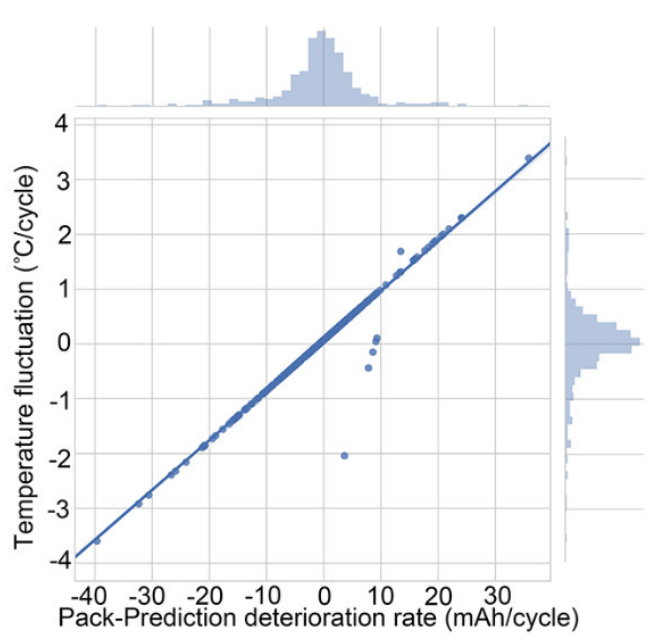

(b)

Figure 17. Correlation between the prediction degradation rate and the temperature fluctuation: (a) cells-prediction; (b) pack-prediction.

As shown in Figure 17, there was a strong correlation between the degradation rate and the temperature fluctuation. However, as seen in Figure 5, the environment temperature was not the only parameter that had an effect on the degradation process of the battery. Due to the lack of information from the cells, other uncertain and certain influences were unknown. In Figure 17, compared with pack-prediction, the cells-prediction provided efficient fluctuation information and avoided excessive dependency on the limited input. The pack prediction with adequate information from the cells performed better, while a lack of information brought an exaggerated effect from input variances.

\subsection{Accuracy Analysis}

Based on the previous analysis result in Section 2.3, in order to clarify the evaluation of the effectiveness of the proposed method, the accuracies of multiple predictions are calculated in this section. Further, the differences were still concluded compared with the pack-prediction method. For the stability of the methods, box plots were adopted to assess the accuracy dispersion of many predictions. The box plots mainly focused on the highest and lowest data points, the median, and the first and third quartiles. These indicators are listed in Table 4 as follows. 
Table 4. Indicators of the box plots.

\begin{tabular}{|c|c|}
\hline Index & Definitions \\
\hline The highest datum & $\begin{array}{l}\text { The highest datum still within } 1.5 \mathrm{IQR} \text { (interquartile range) } \\
\text { of the upper quartile }\end{array}$ \\
\hline The lowest datum & The lowest datum still within $1.5 \mathrm{IQR}$ of the lower quartile \\
\hline Median & $\begin{array}{l}\text { The value separating the higher half from the lower half of } \\
\text { a data sample }\end{array}$ \\
\hline The first quartile & $\begin{array}{l}\text { The middle value between the smallest value and the } \\
\text { median of the data set }\end{array}$ \\
\hline The third quartile & $\begin{array}{c}\text { The middle value between the median and the highest } \\
\text { value of the data set }\end{array}$ \\
\hline
\end{tabular}

In Table 4, the term IQR represents the interquartile deviation, which is the difference between the third quartile and first quartile. The error of different scale predictions is compared in Figure 18, and the data are explained in detail in Table 5. The compared scale was selected to be 20,50, 100, and 400 prediction cycles for a fixed time series.

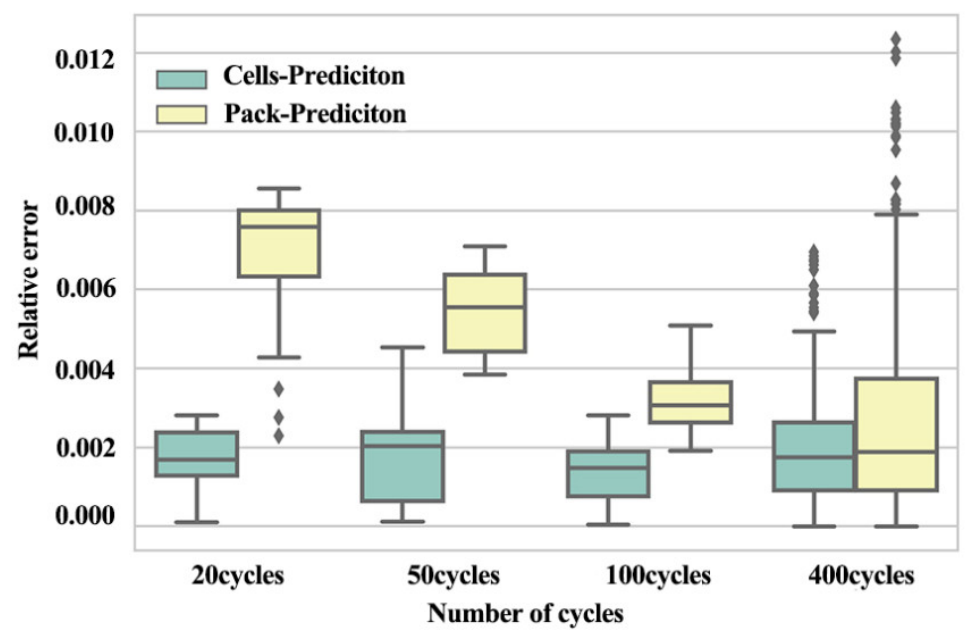

Figure 18. Stability comparison between prediction methods.

Table 5. Mean error of different cycle predictions.

\begin{tabular}{cccccccc}
\hline \multirow{2}{*}{ Methods } & Cycles & $\begin{array}{c}\text { The Highest } \\
\text { Datum }\end{array}$ & $\begin{array}{c}\text { The Third } \\
\text { Quartile }\end{array}$ & Median & $\begin{array}{c}\text { The First } \\
\text { Quartile }\end{array}$ & $\begin{array}{c}\text { The Lowest } \\
\text { Datum }\end{array}$ & Outliers \\
\hline \multirow{3}{*}{ Cells-Prediction } & 20 & 0.002814 & 0.0024 & 0.001699 & 0.001274 & 0.000101 & $0 \%$ \\
\cline { 2 - 8 } & 50 & 0.004534 & 0.002395 & 0.002041 & 0.000443 & 0.000114 & $0 \%$ \\
\hline & 100 & 0.002816 & 0.001903 & 0.001486 & 0.000746 & 0.0000524 & $0 \%$ \\
\hline \multirow{3}{*}{ Pack-Prediction } & 400 & 0004936 & 0.00261 & 0.001718 & 0.00091 & 0.0000406 & $4.08 \%$ \\
\cline { 2 - 8 } & 20 & 0.008563 & 0.008064 & 0.007585 & 0.006075 & 0.0030915 & $15 \%$ \\
\cline { 2 - 8 } & 50 & 0.007093 & 0.006376 & 0.005555 & 0.00439 & 0.00385 & $0 \%$ \\
\hline & 100 & 0.005085 & 0.003641 & 0.003066 & 0.002615 & 0.001921 & $0 \%$ \\
\hline
\end{tabular}

It can be concluded from Figure 18 that there was an obvious disparity between the two prediction methods: 
- The accuracy of the SOH prediction with fused information from the cells was more stable when accompanied by the increase of the prediction cycles. In the pack-prediction method, the accuracy fluctuated too severely to be determined the best prediction scale.

- With the premise that the cells provided sufficient deterioration information, there was less abnormal data for the prediction result. However, with limited input variances in the pack-prediction method, a large number of outliers occurred in the results (as seen in 400 cycles).

- With the increase of the prediction scale, the mean and minimum of the relative errors in the pack-prediction method became better and better. However, in the repeated prediction process, the error fluctuation and abnormal data made it harder to optimize the parameters of the model.

Based on the above analysis, the $\mathrm{SOH}$ performance of the battery packs was significantly improved with the premise of the effective fusion of multi-cell degradation information by LSTM. The main factors were the two aspects that follow.

- The degradation data of the cells provided more trend and fluctuation information for the evolution process of the battery packs, and it could describe the evolution characteristics more accurately. Conversely, the absence of such information meant that the actual $\mathrm{SOH}$ of the cells was not considered, so it would be more difficult to predict and estimate.

- With the premise of using less information (such as using only temperature and time labels as input), it could be observed that in the process of model training the strong effect was produced by the existing limited information (e.g., temperature). The $\mathrm{SOH}$ prediction result of the battery pack only depended on the change of temperature, and the one-sidedness was obviously enhanced. As the number of cells increased, this defect became more severe.

\section{Conclusions}

To solve the problem of insufficient background information from the interaction degradation of cells in $\mathrm{SOH}$ prediction, this paper proposed a modified data-driven method and regulation for the $\mathrm{SOH}$ prediction of a lithium-ion battery pack based on deep learning, with the fusion of multi-cell deterioration information. With experimental data analysis and validation, it was demonstrated that compared to $\mathrm{SOH}$ prediction using only an overall indicator of the packs, the proposed method performed better for future trend forecasting. The proposed method also describes the relationship between degradation process of cells and packs with neural networks. The proposed method mines the deep influence from cells on a battery pack, and improves the input quantity of the information. Because of this, the method improves the prediction accuracy and stability efficiently along with providing internal information on battery pack structures, and prevents the strong dependency on limited external factors.

Take advantages of the development of machine learning, data-driven methods bring a qualitative improvement to $\mathrm{SOH}$ management and prediction in order to help improve the application quality of a power lithium-ion battery. However, in applications, there are still some problems need to solve. On the one hand, even if we proposed ideal model to predict the $\mathrm{SOH}$ of battery packs, the performances of the model still depend on the parameters choices facing to different training data, which is a common problem in machine learning method. On the other hand, the model adopted should be improved and optimized with the development of deep learning technology. More advantageous data-driven analysis methods are preferred to be selected in our future research. What is certain is that according to the ideas and method proposed in this paper, the performance will be gradually optimized with adaptive research carried out in the future.

Author Contributions: X.W. designed the research and experiments; W.F. wrote the paper and analyzed the data; S.L. performed the experiments and polished illustrations; X.L. supervised and administrated the projects; L.W. conceived the research and primary content. All authors have read and agreed to the published version of the manuscript. 
Funding: This research was funded by the National Natural Science Foundation of China (grant number 51805016), Strategic Leading Science \& Technology Programme (Class A) of Chinese Academy of Sciences (grant number XDA14000000), Field Foundation of China (No. JZX7Y20190242012001) and the Fundamental Research Funds for the Central Universities (grant number. YWF-20-BJ-J-908).

Conflicts of Interest: The authors declare no conflict of interest.

\section{References}

1. Zhang, X.; Wang, Y.; Wu, J.; Chen, Z. A novel method for lithium-ion battery state of energy and state of power estimation based on multi-time-scale filter. Appl. Energy 2018, 216, 442-451. [CrossRef]

2. Xiong, R.; Zhang, Y.; He, H.; Zhou, X.; Pecht, M.G. A double-scale, particle-filtering, energy state prediction algorithm for lithium-ion batteries. IEEE Trans. Ind. Electron. 2018, 65, 1526-1538. [CrossRef]

3. Howey, D.A. Tools for battery health diagnostics and prediction. Electrochem. Soc. Interface 2019, $28,55-56$. [CrossRef]

4. Zhang, Y.; Xiong, R.; He, H.; Pecht, M.G. Lithium-ion battery remaining useful life prediction with box-cox transformation and monte carlo simulation. IEEE Trans. Ind. Electron. 2019, 66, 1585-1597. [CrossRef]

5. Wu, B.; Yufit, V.; Marinescu, M.; Offer, G.J.; Martinez-Botas, R.F.; Brandon, N.P. Coupled thermalelectrochemical modelling of uneven heat generation in lithium-ion battery packs. J. Power Sources 2013, 243, 544-554. [CrossRef]

6. Ashwin, T.R.; McGordon, A.; Jennings, P.A. Electrochemical modelling of li-ion battery pack with constant voltage cycling. J. Power Sources 2017, 341, 327-339. [CrossRef]

7. Li, X.; Wang, Z.; Yan, J. Prognostic health condition for lithium battery using the partial incremental capacity and gaussian process regression. J. Power Sources 2019, 421, 56-67. [CrossRef]

8. Ren, H.B.; Zhao, Y.Z.; Chen, S.Z.; Wang, T.P. Design and implementation of a battery management system with active charge balance based on the soc and soh online estimation. Energy 2019, 166, 908-917. [CrossRef]

9. Wang, X.H.; Wang, Z.; Wang, L.Z.; Wang, Z.Q.; Guo, H.Z. Dependency analysis and degradation process-dependent modeling of lithium-ion battery packs. J. Power Sources 2019, 414, 318-326. [CrossRef]

10. Li, J.L.; Zhang, X.R.; Zhou, X.; Lu, L.Y. Reliability assessment of wind turbine bearing based on the degradation-hidden-markov model. Renew. Energy 2019, 132, 1076-1087.

11. Mensi, W.; Hammoudeh, S.; Shahzad, S.J.H.; Al-Yahyaee, K.H.; Shahbaz, M. Oil and foreign exchange market tail dependence and risk spillovers for mena, emerging and developed countries: Vmd decomposition based copulas. Energy Econ. 2017, 67, 476-495. [CrossRef]

12. D'Amico, G.; Petroni, F. Copula based multivariate semi-markov models with applications in high-frequency finance. Eur. J. Oper. Res. 2018, 267, 765-777. [CrossRef]

13. Assaf, R.; Do, P.; Neftimeziani, S.; Scarf, P. Wear rate-state interactions within a multi-component system: A study of a gearbox accelerated life testing platform. Proc. Inst. Mech. Eng. Part O J. Risk Reliab. 2018, 232, 425-434. [CrossRef]

14. Lalropuia, K.C.; Gupta, V. Modeling cyber-physical attacks based on stochastic game and markov processes. Reliab. Eng. Syst. Saf. 2019, 181, 28-37. [CrossRef]

15. Vazquez-Canteli, J.R.; Nagy, Z. Reinforcement learning for demand response: A review of algorithms and modeling techniques. Appl. Energy 2019, 235, 1072-1089. [CrossRef]

16. Goh, T.; Park, M.; Seo, M.; Kim, J.G.; Kim, S.W. Successive-approximation algorithm for estimating capacity of li-ion batteries. Energy 2018, 159, 61-73. [CrossRef]

17. Wei, J.; Dong, G.; Chen, Z. Remaining useful life prediction and state of health diagnosis for lithium-ion batteries using particle filter and support vector regression. IEEE Trans. Ind. Electron. 2018, 65, 5634-5643. [CrossRef]

18. Zhang, Y.; Xiong, R.; He, H.; Pecht, M. Long short-term memory recurrent neural network for remaining useful life prediction of lithium-ion batteries. IEEE Trans. Veh. Technol. 2018, 67, 5695-5705. [CrossRef]

19. Vatani, M.; Vie, P.J.; Ulleberg, Ø. Cycling Lifetime Prediction Model for Lithium-Ion Batteries Based on Artificial Neural Networks. In Proceedings of the 2018 IEEE PES Innovative Smart Grid Technologies Conference Europe (ISGT-Europe), Sarajevo, Bosnia and Herzegovina, 21-25 October 2018; IEEE: Piscataway, NJ, USA, 2018; pp. 1-6. 
20. Mirzapour, F.; Lakzaei, M.; Varamini, G.; Teimourian, M.; Ghadimi, N. A new prediction model of battery and wind-solar output in hybrid power system. J. Ambient Intell. Humaniz. Comput. 2019, 10, 77-87. [CrossRef]

21. Lecun, Y.; Bengio, Y.; Hinton, G. Deep learning. Nature 2015, 521, 436. [CrossRef]

22. Titos, M.; Bueno, A.; Garcia, L.; Benitez, M.C.; Ibanez, J. Detection and classification of continuous volcano-seismic signals with recurrent neural networks. IEEE Trans. Geosci. Remote Sens. 2019, 57, 1936-1948. [CrossRef]

23. Bektas, O.; Jones, J.A.; Sankararaman, S.; Roychoudhury, I.; Goebel, K. A neural network filtering approach for similarity-based remaining useful life estimation. Int. J. Adv. Manuf. Technol. 2019, 101, 87-103. [CrossRef]

24. Zhang, J.; Lee, J. A review on prognostics and health monitoring of li-ion battery. J. Power Sources 2011, 196, 6007-6014. [CrossRef]

25. Lu, L.; Han, X.; Li, J.; Hua, J.; Ouyang, M. A review on the key issues for lithium-ion battery management in electric vehicles. J. Power Sources 2013, 226, 272-288. [CrossRef]

26. Goodfellow, I.A. Deep Learning: Systemy Uczace sięian Goodfellow, Yoshua Bengio, Aaron Courville; Przektad Witkom Witold Sikorski; Wydawnictwo Naukowe PWN: Warsaw, Poland, 2018.

27. Schmidhuber, S.H.J. Long-short term memory. Neural Comput. 1997, 9, 1735-1780.

28. Srivastava, N.; Hinton, G.; Krizhevsky, A.; Sutskever, I.; Salakhutdinov, R. Dropout: A simple way to prevent neural networks from overfitting. J. Mach. Learn. Res. 2014, 15, 1929-1958.

29. Tieleman, T.; Hinton, G. Lecture 6.5-rmsprop: Divide the gradient by a running average of its recent magnitude. Coursera Neural Netw. Mach. Learn. 2012, 4, 26-31.

30. Kurbiel, T.; Khaleghian, S. Training of deep neural networks based on distance measures using rmsprop. arXiv 2017, arXiv:1708.01911.

(C) 2020 by the authors. Licensee MDPI, Basel, Switzerland. This article is an open access article distributed under the terms and conditions of the Creative Commons Attribution (CC BY) license (http://creativecommons.org/licenses/by/4.0/). 